\title{
Constituintes químicos das folhas de Murraya paniculata (Rutaceae)
}

\author{
Sumaia G. Mesquita, ${ }^{1}$ Marcela F. Martinez, ${ }^{1}$ Paulete Romoff, ${ }^{1}$ Oriana A. Fávero, ${ }^{2}$ Sofia $R$. \\ Lieber, ${ }^{2}$ João Henrique G. Lago*,1 \\ ${ }^{1}$ Centro de Ciências e Humanidades, Universidade Presbiteriana Mackenzie, 01302-907 \\ São Paulo-SP, Brasil, \\ ${ }^{2}$ Centro de Ciências Biológicas e da Saúde, Universidade Presbiteriana Mackenzie, 01302-907 \\ São Paulo-SP, Brasil
}

\begin{abstract}
RESUMO: A separação cromatográfica do extrato hexânico e da fase em $\mathrm{CH}_{2} \mathrm{Cl}_{2}$ do extrato etanólico das folhas de Murraya paniculata resultou no isolamento de um triterpeno (24-metileno-cicloartan-3 $\beta$-ol), um fenilpropanóide (cafeato de metila) e sete cumarinas preniladas [isomeranzina, acetato de murranganona, murrayatina, murrangatina, hidrato de meranzina, febalosina e murranganona]. Dentre as substâncias isoladas, as cumarinas foram detectadas anteriormente em M. paniculata ao passo que 24-metileno-cicloartan-3 $\beta$-ol e cafeato de metila estão sendo descritos pela primeira vez no gênero Murraya. Os extratos e frações além das substâncias puras foram submetidos à avaliação do potencial antimicrobiano frente à Staphylococcus aureus e Escherichia coli indicando que somente a cumarina hidrato de meranzina mostrou fraca atividade.
\end{abstract}

Unitermos: Murraya paniculata, cumarinas, terpenóide, fenilpropanóide, atividade antimicrobiana.

\begin{abstract}
Chemical constituents from leaves of Murraya paniculata (Rutaceae)". Chromatographic separation of the hexane extract and the $\mathrm{CH}_{2} \mathrm{Cl}_{2}$ phase from the ethanol extract from leaves of Murraya paniculata yielded one triterpenoid (24-methylene-cycloartan-3 $\beta$-ol), one phenylpropanoid (methyl caffeate) and seven coumarins [isomeranzine, murranganone acetate, murrayatine, murrangatine, meranzine hydrate, phebalosine and murranganone]. All the isolated coumarins were previously obtained from M. paniculata while 24-methylene-cycloartan$3 \beta$-ol and methyl caffeate have been described for first time in the Murraya genus. The crude extracts, fractions and pure substances were submitted to evaluation of antimicrobial potential against Staphylococcus aureus and Escherichia coli which indicated that the coumarin meranzine hydrate showed weak activity.
\end{abstract}

Keywords: Murraya paniculata, coumarins, terpenoid, phenylpropanoid, antimicrobial activity.

\section{INTRODUÇÃO}

Murraya paniculata (sinonímia Murraya exotica), pertencente à família Rutaceae, é uma árvore nativa da Índia que foi introduzida no Brasil, sendo largamente utilizada em arborização e em jardins da cidade de São Paulo. Esta espécie é considerada medicinal nas regiões tropicais e subtropicais da Ásia e também na China e Indonésia. Nesses países, as folhas e raízes são utilizadas para o tratamento de problemas intestinais, de reumatismo e tosse (Velozo, 1995; Lorenzi et al., 2003).

Do ponto de vista químico, esta espécie acumula principalmente cumarinas preniladas e flavonóides, além de derivados do ácido cinâmico e alcalóides (Ito \& Furukawa, 1987; Rahman et al., 1997; Wu et al., 1994; Ferracin et al., 1999). Porém, uma análise dos trabalhos publicados na literatura indica que existe uma variação química significativa entre espécies de diferentes localidades. Assim, em vista da versatilidade de constituíntes químicos e potencial biológico desta espécie vegetal, neste trabalho foi realizada a análise fitoquímica do extrato hexânico e da fase em $\mathrm{CH}_{2} \mathrm{Cl}_{2}$ do extrato etanólico das folhas de Murraya paniculata, os quais após procedimentos cromatográficos forneceram um triterpeno (24-metileno-cicloartan-3 $\beta$-ol -1), um fenilpropanóide (cafeato de metila - 2) e sete cumarinas preniladas [isomeranzina - 3, 7-metoxi-8-(1'-acetoxi2'-oxo-3'-metilbutil)-cumarina - 4, murrayatina - 5, murrangatina - 6, hidrato de meranzina - 7, febalosina - 8 e murranganona - 9]. A identificação estrutural desses compostos foi realizada, principalmente, por análises espectroscópicas de $\mathrm{RMN}$ de ${ }^{1} \mathrm{H}$, de ${ }^{13} \mathrm{C}$ e EM. Adicionalmente, neste trabalho foi avaliada a 
atividade antibacteriana frente à Staphylococcus aureus e Escherichia coli dos extratos, frações e substâncias puras obtidas do fracionamento cromatográfico.

\section{MATERIAL E MÉTODOS}

\section{Procedimentos gerais}

Os espectros de massas (EM) foram registrados em espectros INCOS SO Finnigan - Mat (quadrupolo) operando a impacto eletrônico (IE) a $70 \mathrm{eV}$ acoplado ao cromatográfico 3400 Varian, equipado com coluna capilar DB5 de $30 \mathrm{~m}$ de comprimento e $0,25 \mathrm{~mm}$ de diâmetro. As condições empregadas nos experimentos foram: injetor automático $\left(180{ }^{\circ} \mathrm{C}\right)$, detector por ionização de chama $\left(260{ }^{\circ} \mathrm{C}\right)$, gás carregador $(\mathrm{He})$, vazão $6 \mathrm{~mL} / \mathrm{min}\left(30^{\circ} \mathrm{C}\right)$, temperaturas programadas 100 ${ }^{\circ} \mathrm{C}(2 \mathrm{~min})-100-240{ }^{\circ} \mathrm{C}\left(5{ }^{\circ} \mathrm{C} / \mathrm{min}\right)-240{ }^{\circ} \mathrm{C}(5 \mathrm{~min})$. Os espectros de RMN foram obtidos em espectrômetro Varian Advance operando a $300 \mathrm{MHz}$ para o núcleo de ${ }^{1} \mathrm{H}$ e a $75 \mathrm{MHz}$ para o núcleo de ${ }^{13} \mathrm{C}$, utilizando-se $\mathrm{CDCl}_{3}$ como solvente e TMS (Merck) como padrão de referência interna.

As cromatografias em coluna foram realizadas com sílica gel 70-230 Mesh (Merck) e Sephadex LH-20 (Amersham) enquanto que para as de camada delgada comparativa foram utilizadas placas de gel de sílica (Merck Art. 1.05554) utilizando reveladores como luz ultravioleta (UV) e $\mathrm{H}_{2} \mathrm{SO}_{4}-6 \mathrm{M}$, seguido de aquecimento. Para separações por cromatografia de camada delgada preparativa foram confeccionadas placas de gel de sílica $\mathrm{PF}_{254}$ (Merck Art. 1.07730) de 1,0 mm de espessura.

\section{Material vegetal}

As folhas de Murraya paniculata foram coletadas no Campus da Universidade Presbiteriana Mackenzie, na cidade de São Paulo, em 31 de janeiro de 2005. A espécie foi identificada pela Profa. Oriana A. Fávero e uma exsicata foi depositada no Herbário da Prefeitura Municipal de São Paulo sob número 8762.

\section{Extração e isolamento}

As folhas secas de Murraya paniculata (280 g) foram extraídas com hexano até esgotamento gerando, após eliminação do solvente sob vácuo, $7 \mathrm{~g}$ de extrato hexânico bruto $(\mathrm{H})$. A seguir, a torta foi submetida à extração com EtOH até esgotamento. Após evaporação total do solvente, em sistema de vácuo, foram obtidos 13 $\mathrm{g}$ de extrato etanólico bruto (E). Esse extrato suspendido em EtOH: $\mathrm{H}_{2} \mathrm{O}$ 7:3 e então extraído com hexano (2 L), $\mathrm{CH}_{2} \mathrm{Cl}_{2}(2 \mathrm{~L})$, AcOEt $(2 \mathrm{~L})$ e n-BuOH $(1 \mathrm{~L})$. Após evaporação dos respectivos solventes, foram obtidas $4 \mathrm{~g}$ da fase hexânica (FH), $5 \mathrm{~g}$ da fase em $\mathrm{CH}_{2} \mathrm{Cl}_{2}$ (FD), $2 \mathrm{~g}$ da fase em AcOEt (FA) e $1 \mathrm{~g}$ da fase em n-BuOH (FB).

Uma pequena porção do extrato hexânico bruto
(H - 1g) foi submetida à cromatografia em coluna de gel de sílica sendo o sistema eluído com misturas de $\mathrm{CH}_{2} \mathrm{Cl}_{2} / \mathrm{AcOEt}$ em gradiente crescente de polaridade. Deste processo foram obtidos 14 grupos (GH-1 - GH14). O grupo GH-2 (102 mg) foi submetido à coluna de gel de sílica eluída com uma mistura de hexano/AcOEt em gradiente de polaridade fornecendo 6 subgrupos (GH-2/1 - GH-2/6). O subgrupo GH-2/4 (92 mg) foi purificado por CCDP $\left(\mathrm{CH}_{2} \mathrm{Cl}_{2} / \mathrm{AcOEt} 8: 2\right)$ fornecendo $6 \mathrm{mg}$ de 1. A análise dos espectros de $\mathrm{RMN}$ de ${ }^{1} \mathrm{H}$ e de ${ }^{13} \mathrm{C}$ dos grupos $\mathrm{GH}-4$ e $\mathrm{GH}-5$ mostrou que as mesmas eram constituídas exclusivamente por $8(223 \mathrm{mg})$. O grupo GH-7 (282 mg) foi fracionado em coluna de gel de sílica $\left(\mathrm{CH}_{2} \mathrm{Cl}_{2} / \mathrm{AcOEt}\right.$ em gradiente de polaridade $)$ gerando 7 subgrupos (GH-7/1 - GH-7/7). A substância 7 (90 mg) foi obtida, na forma pura, do subgrupo GH7/4. As frações $\mathrm{GH}-9$ e $\mathrm{GH}-10$ foram reunidas $(45 \mathrm{mg})$ e submetidas à $\mathrm{CCDP}\left(\mathrm{CH}_{2} \mathrm{Cl}_{2} / \mathrm{AcOEt} 7: 3\right)$ fornecendo 7 frações (GH-9+10/1 - GH-9+10/7). A fração GH-9+10/4 mostrou-se constituída pela substância $9(6 \mathrm{mg})$.

A fase em $\mathrm{CH}_{2} \mathrm{Cl}_{2}(\mathrm{FD}-5 \mathrm{~g})$ foi submetida à cromatografia em coluna de gel de sílica sendo o sistema eluido com misturas de hexano/AcOEt e AcOEt/MeOH em polaridade crescente. Deste processo foram obtidos 14 grupos (FD-1 - FD-14). As análises espectrais dos grupos FD-1 (151 mg) e FD-2 (211 mg) mostraram que os mesmos eram compostos basicamente por material graxo. O grupo FD-3 (203 mg) foi submetido à permeação em gel de Sephadex LH-20 (hexano/ $\mathrm{CH}_{2} \mathrm{Cl}_{2}$ 1:4 e $\mathrm{CH}_{2} \mathrm{Cl}_{2} / \mathrm{Me}_{2} \mathrm{CO} 3: 2$ ) do qual foram obtidos 7 subgrupos (FD-3/1 - FD-3/7) das quais FD-3/6 (12 $\mathrm{mg}$ ) mostrou-se constituído por 2. O grupo FD-4 (174 $\mathrm{mg}$ ) foi submetido a fracionamento em coluna de gel de sílica eluida com hexano/AcOEt em gradiente de polaridade. Deste processo foram obtidos 9 subgrupos (FD-4/1 - FD-4/9) sendo a substância 3 (5 mg) isolada de FD-4/9. O grupo FD-5 (759 mg) foi separado em coluna de gel de sílica eluída com misturas de hexano/AcOEt em gradiente de polaridade. Esse procedimento resultou na obtenção de 13 subgrupos (FD-5/1 - FD-5/13). Os subgrupos FD-5/6, FD-5/7 e FD-5/8 foram reunidos (46 $\mathrm{mg}$ ) e então submetidos à purificação através de CCDP (hexano/AcOEt 3:7) sendo obtidos $7 \mathrm{mg}$ de 4 e $13 \mathrm{mg}$ de 5. O grupo FD-7 $(1,0 \mathrm{~g})$ foi submetido à separação em coluna de gel de sílica, eluída com misturas de hexano/ AcOEt em gradiente de polaridade, e posteriormente com AcOEt/MeOH 1:1 da qual foram obtidos 9 subgrupos (FD-7/1 - FD-7/9). Os subgrupos FD-7/3 e FD-7/4 foram reunidos $(512 \mathrm{mg})$ e submetidos à permeação em gel de Sephadex LH-20 (MeOH puro) sendo obtidas 4 frações (FD-7/3+4/1 - FD-7/3+4/4). A fração FD$7 / 3+4 / 2(139 \mathrm{mg})$ foi purificada por CCDP (AcOEt puro) fornecendo $5 \mathrm{mg}$ de 6 . O grupo FD-8 (460 mg) foi submetido à permeação em gel de Sephadex LH-20 (hexano/ $\mathrm{CH}_{2} \mathrm{Cl}_{2} 1: 4$ e $\mathrm{CH}_{2} \mathrm{Cl}_{2} / \mathrm{Me}_{2} \mathrm{CO} 3: 2$ ) fornecendo 9 subgrupos (FD-8/1 - FD-8/9). Os subgrupos FD-8/6 (149 mg) e FD-8/7 (19 mg) mostraram-se constituídos 
por 7 e 6, respectivamente.

\section{Ensaios biológicos}

\section{Avaliação da atividade antibacteriana}

A avaliação da atividade antibacteriana dos extratos e frações das folhas de Murraya paniculata foi determinada através do método de difusão em gel (Bauer et al., 1966; Ulubelen et al., 2000; Andrade et al., 2005) pela Prof ${ }^{a}$ Dr $^{a}$ Sofia R. Lieber no Laboratório de Microbiologia do CCBS da Universidade Presbiteriana Mackenzie. Assim, o extrato hexânico (H), as fases em hexano (FH), em $\mathrm{CH}_{2} \mathrm{Cl}_{2}$ (FD), em AcOEt (FA) e em n-BuOH (FB) proveniente da partição do extrato $\mathrm{EtOH}$ bruto foram esterilizados através de filtração em membranas de poro de $0,20 \mu \mathrm{m}$ e em seguida adicionados em discos de papel de filtro estéreis de 6 mm de diâmetro, na concentração de $1000 \mu \mathrm{g} /$ disco.

Para o ensaio em questão foram utilizadas duas bactérias, uma Gram positiva (Staphylococcus aureus ATCC 6538) e outra Gram negativa (Escherichia coli - ATCC 8739). A avaliação da atividade foi verificada através da presença de uma zona de inibição do crescimento ao redor do disco.

O crescimento bacteriano foi avaliado após 24 horas de incubação da placa a $37{ }^{\circ} \mathrm{C}$. Como controles positivos da inibição foram utilizados discos de gentamicina ( $240 \mu \mathrm{g} /$ disco) além de discos impregnados com os solventes, para verificação do controle de inibição dos mesmos.

\section{RESULTADOS E DISCUSSÃO}

A separação cromatográfica do extrato hexânico e da fase em $\mathrm{CH}_{2} \mathrm{Cl}_{2}$ do extrato etanólico das folhas de Murraya paniculata resultou no isolamento de um triterpeno (1), um fenil propanóide (2) e sete cumarinas preniladas (3-9). Destas, as substâncias 1 e 2 estão sendo descritas pela primeira vez no gênero Murraya enquanto que todas as cumarinas isoladas foram detectadas anteriormente em $M$. paniculata (Ito \& Furukawa, 1987).

$\mathrm{O}$ espectro de RMN de ${ }^{1} \mathrm{H}$ de $\mathbf{1}$ mostra, entre outros, dois dubletos centrados em $\delta 0,33(J=4,2$ $\mathrm{Hz}, 1 \mathrm{H})$ e em $\delta 0,50(J=4,2 \mathrm{~Hz}, 1 \mathrm{H})$, os quais são atribuídos ao H-19 de derivados triterpênicos com esqueleto cicloartano (Teresa et al., 1987; Lago et al., 2002). Esses sinais, associados aqueles característicos de hidrogênios geminados ligados a carbono $\mathrm{sp}^{2} \mathrm{em} \delta$ 4,78 (s, H-31a) e em $\delta 4,63$ (s, H-31b), e referente ao H-3 ligado a carbono carbinólico em $\delta 3,46(\mathrm{sl})$, permite inferir que 1 apresenta a estrutura de um cicloartan-3 $\beta$-ol com uma ligação dupla terminal na cadeia lateral. Para confirmação estrutural, esse composto foi submetido à análise por espectrometria de massas, sendo observado o pico do íon-molecular em $m / z 440 \mathrm{Da}$, compatível com a fórmula molecular $\mathrm{C}_{31} \mathrm{H}_{52} \mathrm{O}$. Finalmente, a análise por $\mathrm{RMN}$ de ${ }^{13} \mathrm{C}$ mostrou 31 sinais sendo aqueles em $\delta 78,8$ (C-3), 156,8 (C-24) e 106,1 (C-31) compatíveis aos carbonos oximetínicos e de ligação dupla terminal, respectivamente. Desta forma, após comparação com os dados espectrais descritos para o 24-metileno-cicloartan$3 \beta$-ol (Teresa et al., 1987), tornou-se possível identificar a estrutura de 1 .

$\mathrm{O}$ espectro de $\mathrm{RMN}$ de ${ }^{1} \mathrm{H}$ de 2 mostra dois dubletos em $\delta 7,59(J=15,9 \mathrm{~Hz}, 1 \mathrm{H})$ e em $\delta 6,25(J=$ $15,9 \mathrm{~Hz}, 1 \mathrm{H})$, correspondentes a hidrogênios de ligação dupla com configuração trans. Além desses sinais, foram observados um dubleto em $\delta 6,88(J=8,1 \mathrm{~Hz}, 1 \mathrm{H})$, um dubleto em $\delta 7,00(J=1,8 \mathrm{~Hz}, 1 \mathrm{H})$ e um duplo-dubleto em $\delta 7,04(J=8,1$ e 1,8 Hz, 1H), referentes à hidrogênios de um sistema aromático 1,3,4-trissubstituído. Este espectro mostra ainda dois singletos em $\delta 3,77(3 \mathrm{H})$ e em $\delta 3,89(3 \mathrm{H})$, referentes a dois grupos metoxílicos. A análise do espectro de massas de 2 mostrou o pico do íon-molecular em $\mathrm{m} / \mathrm{z} 208 \mathrm{Da}$, compatível com a fórmula $\mathrm{C}_{11} \mathrm{H}_{12} \mathrm{O}_{4}$. A comparação dos dados de espectrométricos com aqueles descritos na literatura (Moreira et al., 2000) permitiu a caracterização estrutural de 2 como 4-hidroxi3-metoxi-cinamato de metila, também conhecido como cafeato de metila.

$\mathrm{O}$ espectro de $\mathrm{RMN}$ de ${ }^{1} \mathrm{H}$ de 3 mostrou dois dubletos em $\delta 6,23(J=9,6 \mathrm{~Hz}, 1 \mathrm{H})$ e em $\delta 7,64(J=$ $9,6 \mathrm{~Hz}, 1 \mathrm{H})$, característicos de hidrogênios de ligação dupla de configuração cis além de outros dois dubletos em $\delta 7,38(J=8,7 \mathrm{~Hz}, 1 \mathrm{H})$ e em $\delta 6,85(J=8,7 \mathrm{~Hz}, 1 \mathrm{H})$ relativos a dois hidrogênios vicinais de anel aromático. Esse perfil mostrou-se indicativo da ocorrência de substâncias das classes das cumarinas (Ito \& Furukawa, 1987). Uma vez que foram observados apenas dois sinais referentes aos hidrogênios aromáticos que acoplam entre si, pode-se inferir que está presente, na molécula de $\mathbf{3}$, um substituinte na posição 8 . O espectro de RMN de ${ }^{1} \mathrm{H}$ mostra, além dos sinais descritos acima, um dubleto em $\delta 1,21(J=6,9 \mathrm{~Hz}, 6 \mathrm{H})$ e um hepteto em $\delta 2,82(J=$ $6,9 \mathrm{~Hz}, 1 \mathrm{H})$ que associados a presença de um singleto em $\delta 4,02(2 \mathrm{H})$ sugerem a presença de uma unidade derivada do isopreno em C-8. Além disso, os valores de deslocamento químico dos hidrogênios da cadeia lateral são sugestivos da presença de um grupo carbonílico em C-2' (Silverstein et al., 1991). Finalmente, a estrutura de 3 foi totalmente elucidada pela atribuição do singleto em $\delta 3,87(3 \mathrm{H})$ ao grupo metoxílico ligado ao anel aromático na posição 7 . A confirmação da identidade estrutural de $\mathbf{3}$ foi realizada após análise dos dados do espectro de RMN de ${ }^{13} \mathrm{C}$ (Tabela 1), o qual mostrou treze sinais, dos quais dois relativos aos grupos carbonílicos da cadeia isoprênica em $\delta 210,7$ e do anel lactônico em $\delta 161,0$. Além desses foram observados os sinais do anel aromático e da ligação dupla em $\delta 160,5$ (C), 153, $2(\mathrm{C}), 143,8(\mathrm{CH}), 127,5(\mathrm{CH}), 113,0(\mathrm{CH}), 112,9(\mathrm{C})$, $112,3(\mathrm{C})$ e em $\delta 107,3(\mathrm{CH})$ e dos sinais relativos a cadeia lateral em $\delta 40,9(\mathrm{CH}), 34,7\left(\mathrm{CH}_{2}\right)$ e $\delta 18,4(2$

Rev. Bras. Farmacogn. Braz J. Pharmacogn 18(4): Out./Dez. 2008 
$\mathrm{X}_{\mathrm{CH}_{3}}$ ) e do grupo metoxílico em $\delta 56,1$. Desta forma, através da comparação dos dados de RMN com aqueles descritos na literatura (Ito \& Furukawa, 1987; Abaul et al., 1994), a estrutura de 3 foi definida como 7-metoxi8-(2'-oxo-3'-metilbutil)-cumarina também conhecida como isomeranzina.

$\mathrm{O}$ espectro de RMN de ${ }^{1} \mathrm{H}$ de 4 mostra quatro dubletos em $\delta 6,27(J=9,6 \mathrm{~Hz}, 1 \mathrm{H}), 7,65(J=9,6 \mathrm{~Hz}$, $1 \mathrm{H}), 6,90(J=8,7 \mathrm{~Hz}, 1 \mathrm{H})$ e em $\delta 7,48(J=8,7 \mathrm{~Hz}$, $1 \mathrm{H})$ além de um singleto em $\delta 3,90(3 \mathrm{H})$, semelhantes aqueles observados para a substância 3 . Tal perfil sugere que 4 seja uma cumarina contendo um grupo metoxílico em C-7. Além desses sinais, foi observado neste espectro um singleto em $\delta 2,16(3 \mathrm{H})$, característico de grupo acetila, um hepteto em $\delta 2,80(J=6,7 \mathrm{~Hz}, 1 \mathrm{H})$ e dois dubletos em $\delta 1,18(J=6,7 \mathrm{~Hz}, 3 \mathrm{H})$ e em $\delta 1,03(J=$ $6,7 \mathrm{~Hz}, 3 \mathrm{H})$ os quais foram atribuídos, respectivamente, a H-3', H-4' e H-5'. Após a comparação com dados dos dados de RMN de ${ }^{1} \mathrm{H}$ com aqueles descritos na literatura (Ito \& Furukawa, 1987) a estrutura de 4 foi definida como 7-metoxi-8-(1'-acetoxil-2' -oxo-3'-metilbutil)cumarina, conhecida como acetato de murranganona. Para confirmação da estrutura proposta, foi registrado o espectro de $\mathrm{RMN}$ de ${ }^{13} \mathrm{C}$ onde foram observados, entre outros, sinais relativos à unidade cumarínica em $\delta 161,1$ (C-2), 114,1 (C-3), 143,4 (C-4), 113,4 (C-4a), 130,3 (C-5), 108,1 (C-6), 160,0 (C-7), 114,8 (C-8), 153,0 (C$8 \mathrm{a})$ e $\delta 56,7\left(\mathrm{OCH}_{3}\right)$. Os sinais em $\delta 173,0(\mathrm{C})$ e em $\delta$ $21,0\left(\mathrm{CH}_{3}\right)$ foram atribuídos ao grupamento acetoxílico e aquele em $\delta$ 69,7 (CH), característico de carbono carbinólico, foi atribuído ao carbono C-1'. Além desses, o sinal referente ao carbono carbonílico C-2' foi observado em $\delta 212,1$ e o dos grupos metílicos C-4' e C-5' em $\delta$ 19,2 e 18,3, respectivamente (Tabela 1).

$\mathrm{O}$ espectro de RMN de ${ }^{1} \mathrm{H}$ de 5 mostrou sinais característicos de cumarina 7,8-dissubstituída, como observado para 3 e 4 . Além desses, foram observados dois dubletos em $\delta 0,72(J=6,3 \mathrm{~Hz}, 3 \mathrm{H})$ e em $\delta 0,66$ $(J=6,3 \mathrm{~Hz}, 3 \mathrm{H})$, referentes a dois grupos metílicos ligados a carbono metínico além de dois singletos em $\delta 1,36(3 \mathrm{H})$ e em $\delta 1,32(3 \mathrm{H})$, característicos de grupos metílicos ligados a carbono carbinólico. $\mathrm{O}$ espectro de $\mathrm{RMN}$ de ${ }^{13} \mathrm{C}$ mostrou, entre outros, sinais referentes a carbonos do tipo $\mathrm{sp}^{2}$ (anel aromático e ligação dupla) em $\delta$ 112,9 (C-3), 143,7 (C-4), 112,9 (C-4a), 127,1 (C-5), 107,2 (C-6), 160,0 (C-7), 114,8 (C-8) e 153,0 (C-8a), a carbonos carbinólicos em $\delta 78,3$ (C-2') e $\delta$ 72,6 (C-3') e a quatro grupos metílicos em $\delta 25,1$ (C-4'), 25,2 (C5'), 22,0 (C-4") e em $\delta 22,1$ (C-5"), como mostrado na Tabela 1. Após a comparação dos dados de RMN com aqueles descritos na literatura (Barik et al., 1983; Ito \& Furukawa, 1987) foi possível identificar o composto 5 como 7-metoxi-8-[2'-(3"'-metilbutanoato)-3'-hidroxi3 '-metilbutil]-cumarina, conhecida como murrayatina.

No espectro de $\mathrm{RMN}$ de ${ }^{1} \mathrm{H}$ de $\mathbf{6}$ foram observados sinais característicos de cumarinas C-7 metoxiladas (Ito \& Furukawa, 1987) além de dois dubletos em $\delta 4,67(J=1,8 \mathrm{~Hz}, 1 \mathrm{H})$ e $\delta 4,59(J=1,8 \mathrm{~Hz}$,

Tabela 1. Dados de $\mathrm{RMN}$ de ${ }^{13} \mathrm{C}\left(75 \mathrm{MHz}, \mathrm{CDCl}_{3}\right)$ para as cumarinas 3 - 9.

\begin{tabular}{|c|c|c|c|c|c|c|c|}
\hline posição & 3 & 4 & 5 & 6 & 7 & 8 & 9 \\
\hline 2 & 161,0 & 161,1 & 161,2 & 160,1 & 161,3 & 160,3 & 160,0 \\
\hline 3 & 113,0 & 114,1 & 112,9 & 113,7 & 112,7 & 113,3 & 113,4 \\
\hline 4 & 143,8 & 143,4 & 143,7 & 143,9 & 143,9 & 143,4 & 143,5 \\
\hline $4 a$ & 112,9 & 113,4 & 112,9 & 113,5 & 112,9 & 112,4 & 113,4 \\
\hline 5 & 127,5 & 130,3 & 127,1 & 128,5 & 126,9 & 128,9 & 129,6 \\
\hline 6 & 107,3 & 108,1 & 107,2 & 107,8 & 107,4 & 107,5 & 107,8 \\
\hline 7 & 160,0 & 160,0 & 160,5 & 160,0 & 160,5 & 161,8 & 160,5 \\
\hline 8 & 112,0 & 114,8 & 116,0 & 116,1 & 115,8 & 113,4 & 114,0 \\
\hline $8 \mathrm{a}$ & 153,2 & 153,0 & 153,2 & 152,9 & 153,3 & 153,7 & 153,3 \\
\hline 1 & 34,7 & 69,7 & 23,2 & 69,6 & 25,5 & 51,7 & 68,4 \\
\hline 2 ' & 210,8 & 212,1 & 78,3 & 78,3 & 78,1 & 60,5 & 212,5 \\
\hline 3 & 40,9 & 36,5 & 72,6 & 143,8 & 73,0 & 141,2 & 35,8 \\
\hline $4 '$ & 18,4 & 19,2 & 25,1 & 113,1 & 24,1 & 113,4 & 19,5 \\
\hline 5 & 18,4 & 18,3 & 25,2 & 17,8 & 25,9 & 17,3 & 17,9 \\
\hline $\mathrm{OMe}$ & 56,1 & 56,7 & 56,1 & 56,1 & 56,1 & 56,2 & 56,1 \\
\hline $1 "$ & - & 173,0 & 172,8 & - & - & - & - \\
\hline $2 "$ & - & 21,0 & 43,2 & - & - & - & - \\
\hline $3 "$ & - & - & 26,6 & - & - & - & - \\
\hline 4" & - & - & 22,0 & - & - & - & - \\
\hline $5 "$ & - & - & 22,1 & - & - & - & - \\
\hline
\end{tabular}




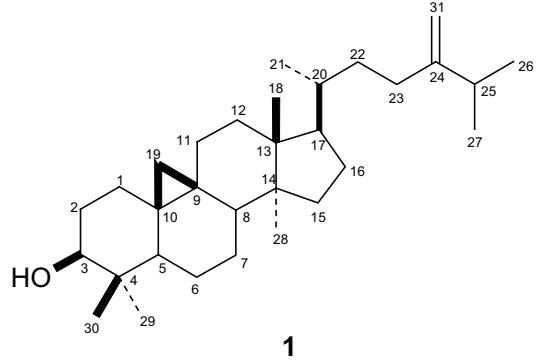<smiles>COC(=O)/C=C/c1ccc(O)c(OC)c1</smiles>

2<smiles>[R]c1c(OC)ccc2ccc(=O)oc12</smiles>

3<smiles></smiles>

$4 \mathrm{R}=$<smiles>[3H]C(OC(C)=O)C(=O)C(C)C</smiles>

$5 \mathrm{R}=$<smiles>CCC(OC(=O)[CH]C(C)C)C(C)(C)O</smiles><smiles>[CH-][C@@H](O)[C@@H](O)C(=C)C</smiles>

$\mathrm{R}=$<smiles>CCC(O)C(C)(C)O</smiles>

$9 \mathrm{R}=$<smiles>[3H]C(O)C(=O)C(C)C</smiles>

1H), característicos de hidrogênios geminados de ligação dupla terminal. Essa observação, associada à presença de um singleto em $\delta 1,79(3 \mathrm{H})$, característico de grupo metílico ligado a carbono $\mathrm{sp}^{2}$, permite inferir a presença de uma unidade 3-metil-3-butenil ligado a C-8. Uma vez que no espectro de RMN de ${ }^{1} \mathrm{H}$ foram observados ainda um dubleto em $\delta 5,33(J=8,7 \mathrm{~Hz}, 1 \mathrm{H})$ e um dubleto em $\delta 4,54(J=8,7 \mathrm{~Hz}, 1 \mathrm{H})$ foi sugerida a presença de dois carbonos carbinólicos vicinais, o que foi confirmado pela observação, no espectro de $\mathrm{RMN}$ de ${ }^{13} \mathrm{C}$, dos sinais em $\delta$ 69,5 e $\delta 78,3$ atribuídos aos carbonos oximetínicos $\mathrm{C}-1$ ' e C-2'. Neste espectro foram ainda observados sinais em $\delta 143,8,113,8$ e 17,3, relativos aos carbonos C-3', C-4' e C-5', respectivamente. Finalmente a comparação dos dados espectrais, principalmente $\mathrm{RMN}$ de ${ }^{13} \mathrm{C}$, com aqueles descritos na literatura (Ito \& Furukawa, 1987; Kinoshita et al., 1996) para 7-metoxi-8-(1',2'diidroxi-3'-metilbut-3'-enil)-cumarina, conhecida como murrangatina, permitiu a identificação de 6 .

O espectro de $\mathrm{RMN}$ de ${ }^{1} \mathrm{H}$ de 7 mostrou, além dos sinais referentes a unidade cumarínica, um singleto em $\delta$ $1,31(6 \mathrm{H})$, referente a dois grupos metílicos equivalentes ligados à carbono carbinólico. Esse sinal, associado à presença de um duplo-dubleto em $\delta 3,61(J=8,9$ e $3,9 \mathrm{~Hz}, 1 \mathrm{H})$, indicativo de hidrogênio ligado a carbono oximetínico, e um multipleto em $\delta 3,02(2 \mathrm{H})$, sugere a presença de uma unidade isoprênica 2,3-diidroxilada. $\mathrm{O}$ espectro de $\mathrm{RMN}$ de ${ }^{13} \mathrm{C}$ mostrou quinze sinais, dentre os quais dois carbonos carbinólicos em $\delta 78,1$ (C-2') e em $\delta 73,0$ (C-3') e três carbonos alifáticos em $\delta 25,5$ (C-1'), $\delta 24,1$ (C-4') e $\delta 25,9$ (C-5') o que confirma a presença do grupo 2',3'-diidroxi-isoprênico, além de sinais referentes aos carbonos da unidade cumarínica, os quais foram atribuídos tendo como modelos os compostos 3 - 6 (Tabela 1). A comparação dos dados de RMN com aqueles descritos na literatura (Ito \& Furukawa, 1987; Dondon et al., 2006) permitiu a identificação de 7 como 7-metoxi-8-(1',2'-diidroxi-3'-metilbutil)-cumarina, conhecida como hidrato de meranzina.

$\mathrm{O}$ espectro de RMN de ${ }^{1} \mathrm{H}$ de 8 mostrou grande semelhança com aquele observado para $\mathbf{6}$, inclusive pela presença de um singleto em $\delta 1,83(3 \mathrm{H})$, referente a hidrogênios metílicos ligados a carbono $\mathrm{sp}^{2}$, de dois dubletos em $\delta 5,04(J=1,7 \mathrm{~Hz}, 1 \mathrm{H})$ e em $\delta 5,27(J=1,7$ $\mathrm{Hz}, 1 \mathrm{H})$, relativos a hidrogênios de carbono metilênico terminal e de dois dubletos em $\delta 3,95(J=2,4 \mathrm{~Hz}$, $1 \mathrm{H})$ e em $\delta 3,87(J=2,4 \mathrm{~Hz}, 1 \mathrm{H})$, cujos valores de deslocamento químico são indicativos de hidrogênios ligados a carbonos oximetínicos de epóxido (Pohlit \& Ferraz, 1995). O espectro de massas de 8 mostrou o íonmolecular em $m / z 258 \mathrm{Da}$, compatível com a fórmula molecular $\mathrm{C}_{15} \mathrm{H}_{14} \mathrm{O}_{4}$, com nove graus de insaturação, sugerindo de um anel epóxido na cadeia lateral. Tais dados sugerem a ocorrência da cumarina febasolina, cuja estrutura foi definida após comparação dos dados de $\mathrm{RMN}$ de ${ }^{1} \mathrm{H}$ e de EM com aqueles descritos na literatura (Ito \& Furukawa, 1987). A análise do espectro de $\mathrm{RMN}$ de ${ }^{13} \mathrm{C}$ permitiu confirmar a estrutura de 8 devido aos nove sinais referentes a unidade cumarínica (entre $\delta 161,8$ - 107,5), ao grupo metoxílico ligado a C-7 ( $\delta 56,2)$, aos carbonos sp ${ }^{2}$ C-3' $(\delta 141,2)$ e C-4' $(\delta$ $113,4)$, ao grupo metílico $C-5^{\prime}$ ' $(\delta 17,3)$, e aos carbonos carbinólicos de epóxido C-1' $(\delta 51,7)$ e C-2' $(\delta 60,5)$, como mostrado na Tabela 1.

Além dos sinais referentes à unidade cumarínica, no espectro de RMN de ${ }^{1} \mathrm{H}$ de 9 foram observados dois dubletos em $\delta 1,06(J=6,9 \mathrm{~Hz}, 3 \mathrm{H})$ e em $\delta 1,00(J=6,9$ $\mathrm{Hz}, 3 \mathrm{H})$, os quais podem ser atribuídos a dois grupos metílicos de uma unidade isoprênica, ou seja, a H-4' e 
H-5'. Ainda nesse espectro, foi observado um singleto largo em $\delta 5,92(1 \mathrm{H})$, que devido à desproteção observada, foi atribuído ao hidrogênio ligado a C-1', carbinólico. Uma vez que não foram observados outros sinais no espectro de RMN de ${ }^{1} \mathrm{H}$, os quais possam ser atribuídos a H-2', foi sugerido o carbono C-2' é carbonílico. Para confirmação estrutural, a substância 9 foi analisada por espectrometria de massas, a qual mostrou, para esse derivado, o pico do íon molecular em $\mathrm{m} / \mathrm{z} 276$ $\mathrm{Da}$, compatível com a fórmula molecular $\mathrm{C}_{15} \mathrm{H}_{16} \mathrm{O}_{5}$, com oito insaturações. Destas, quatro são referentes ao anel aromático, três à lactona $\alpha, \beta$-insaturada e uma à carbonila da cadeia lateral. Além disso, neste espectro foi observado o pico base em $\mathrm{m} / z$ 205, compatível com o fragmento $\left[\mathrm{C}_{11} \mathrm{H}_{9} \mathrm{O}_{4}\right]^{+}$, relativo a clivagem da ligação C-1'/C-2' somente possível pela presença do grupo carbonila em C-2' na cadeia isoprênica. Finalmente, a estrutura de $\mathbf{9}$ foi confirmada pela análise dos dados de $\mathrm{RMN}$ de ${ }^{13} \mathrm{C}$ (Tabela 1), principalmente pelos sinais referentes aos carbonos da cadeia lateral em $\delta 68,4 \mathrm{e}$ em $\delta$ 212,5 atribuídos a C-1' e C-2', respectivamente, e por comparação com os dados espectrais com aqueles descritos na literatura (Ito \& Furukawa, 1987; Imai et al., 1989) para 7-metoxi-8-(1'-hidroxi-2'-oxo-3'metilbutil)-cumarina, conhecida como murranganona.

$\mathrm{Na}$ avaliação da atividade antibacteriana dos extratos hexânico $(\mathrm{H})$ e etanólico (E) assim como das fases de partição desse último (FH, FD, FA e FB) foi observado que o extrato hexânico bruto $(\mathrm{H})$ e a fase em diclorometano (FD) apresentaram atividade antibacteriana frente a $S$. aureus. Após separação dos principais componentes dessas fases, estes foram avaliados quanto ao potencial antimicrobiano tendo sido observado que somente a substância 7 apresentou fraca atividade (halo de inibição $=11 \mathrm{~mm}-1000 \mu \mathrm{g} /$ disco) em relação ao controle positivo - gentamicina (halo de inibição $=27 \mathrm{~mm}-240 \mu \mathrm{g} /$ disco) ao passo que as substâncias restantes foram inativas neste ensaio.

\section{AGRADECIMENTOS}

Os autores agradecem as agencias de fomento (MackPesquisa e FAPESP) pelo auxílio concedido para realização desse trabalho.

\section{REFERENCIAS}

Abaul J, Philogène E, Bourgeois, Poupat C, Ahond A, Potier P 1994. Contribution à la connaissance des Rutacées Américaines: étude des feuilles de Triphasia trifolia. J Nat Prod 57: 846-848.

Andrade CA, Peitz C, Cúnico M, Carvalho JLS, Abrahão WM, Miguel OG, Miguel MD, Kerber VA 2005. Avaliação da atividade antibacteriana e triagem fitoquímica das flores de Acacia podalyriifolia A. Cunn. Ex G. Don Leguminosae-Mimosoideae. Rev Bras Farmacogn 15: 13-15.

Barik BR, Dey AK, Chatterjee A 1983. Murrayatin, a coumarins from Murraya exotica. Phytochemistry 22: 2273-2275

Bauer AW, Kirby WM, Sherris JC, Turck M 1966. Antibiotic susceptibility testing by standardized single disc method. Am J Clin Patol 45: 493-496.

Dondon R, Bourgeois P, Fery-Forgues S 2006. A new bicoumarin from the leaves and stems of Triphasia trifolia. Fitoterapia 77: 129-133.

Ferracin RJ, da Silva MFGF, Fernandes JB, Vieira PC 1999. Flavonoids from the fruits of Murraya paniculata. Phytochemistry 47: 393-396.

Imai F, Kinoshita T, Sankawa U 1989. Constituents of the leaves of Murraya paniculata collected in Taiwan. Chem Pharm Bull 37: 358-362.

Ito C, Furukawa H 1987. Constituents of Murraya exotica L. Structure elucidation of new coumarins. Chem Pharm Bull 35: 4277-4285.

Kinoshita T, Wu JB, Ho FC 1996. The isolation of a prenylcoumarin of chemotaxonomic significance from Murraya paniculata var. omphalocarpa. Phytochemistry 43: 125-128.

Lago JHG, Roque NF 2002. Cycloartane triterpenoids from Guarea macrophylla. Phytochemistry 60: 329-332.

Lorenzi H, Souza HM, Torres MAV, Bacher LB 2003. Árvores Exóticas no Brasil, madeireiras, ornamentais e aromáticas. Nova Odessa/SP: Instituto Plantarum de Estudos da Flora, p 332.

Moreira DL, Guimarães EF, Kaplan MAC 2000. A C-glucosylflavone from leaves of Piper lhotzkyanum. Phytochemistry 55: 783-786.

Pohlit AM, Ferraz HMC 1995. Estudos de RMN de epóxidos: Uma visão geral. Quim Nova 18: 160-170.

Rahman AU, Shabber M, Sultani SZ, Jabbar A, Choudhary MI 1997. Cinnamates and coumarins from the leaves of Murraya paniculata. Phytochemistry 44: 483-485.

Silverstein RM, Bassler GC, Morril TC 1991. Spectrometric Identification of Organic Compounds. New York: John Wiley \& Sons, Inc., $5^{\text {th }}$ Edition, $\mathrm{p} 212$.

Teresa JP, Urone JG, Marcos JS, Basabe P, Cuadrado MJS, Moro RF 1987. Triterpenes from Euphorbia broteri. Phytochemistry 26: 1767-1776.

Ulubelen A, Öksüz S, Bozok-Johansson C, Celik C, Voelter W 2000. Antibacterial diterpenes from the roots of Salvia viridis. Planta Med 66: 458-462.

Velozo ES 1995. Fitoquímica comparada dos gêneros Angostura, Almeidea e Rauia (Rutaceae). São Carlos, 127 p. Tese de Doutorado - Programa de Pósgraduação em Química, Universidade Federal de São Carlos.

Wu TS, Chan YY, Leu YL, Huang SC 1994. A flavonoid and indole alkaloid from flowers of Murraya paniculata. Phytochemistry 37: 287-288. 